\title{
Flow pumping system for physiological waveforms
}

\author{
William Tsai • Ömer Savaş
}

Received: 27 July 2009/ Accepted: 8 December 2009/Published online: 6 January 2010

(c) The Author(s) 2010. This article is published with open access at Springerlink.com

\begin{abstract}
A pulsatile flow pumping system is developed to replicate flow waveforms with reasonable accuracy for experiments simulating physiological blood flows at numerous points in the body. The system divides the task of flow waveform generation between two pumps: a gear pump generates the mean component and a piston pump generates the oscillatory component. The system is driven by two programmable servo controllers. The frequency response of the system is used to characterize its operation. The system has been successfully tested in vascular flow experiments where sinusoidal, carotid, and coronary flow waveforms are replicated.
\end{abstract}

Keywords Pulsatile flow pump - Physiological waveform · Blood flow · Gear-piston pumping system . Waveform replication

\section{Introduction}

It is essential to simulate the pulsatile nature of blood flow when examining the fluid mechanics of the human circulatory system. Flow waveforms at numerous locations in the body have been measured in vivo using a variety of methods, such as magnetic resonance imaging (MRI) and Doppler ultrasound. This has allowed the design of pumps capable of replicating physiological flow conditions in vitro.

One approach is to develop systems that replicate the heart's pumping mechanism and build a circulatory model around it. Segers et al. [13] created a pump that replicated

W. Tsai $(\bowtie) \cdot$ Ö. Savaş

Department of Mechanical Engineering, University

of California, Berkeley, Berkeley, CA 94720, USA

e-mail: wtsai@berkeley.edu the mechanical action of the left atrium, ventricle, and valves. The pump was attached to a replica arterial tree using a network of branching elastic tubes. The flow measurements from the system matched physiological measurements at various points of the arterial network. A commercially available cardiac replication pump (Vivtro Labs, Victoria, BC, Canada) is capable of cardiac output simulation [7, 12]. The system consists of a linear actuatorpiston combination that replicates an input waveform. The pump drives the motion of a compliant elastic model of the heart, simulating cardiac output flow. Replicating a specific arterial waveform requires adjusting the impedance of the flow path.

Rather than replicating flow from the heart to the desired artery, an alternate method is used to generate a measured flow waveform for a specific artery. A commercially available, dual-chamber pump (Shelly Imaging Systems, London, ON, Canada) uses a reversing cylinder-piston to generate pulsatile flow $[2,3]$. The piston divides the cylinder into two chambers, each with fluid ports at the end. As the piston is driven in one direction, the pulsatile waveform is output from a chamber while its compliment is refilled from the return line. Once the piston has been driven to one end of the cylinder, the piston reverses and the sequence repeats. All fluid ports connect to a computercontrolled 4-way valve which sequences the flow directions. Law et al. [8] developed a modified roller pump connected to a computer-controlled stepper motor for waveform replication, which offers satisfactory waveform replication but is limited by the diameter of the tubing in the pump. Eriksson et al. [1] developed a pump that used a computer-controlled progressive cavity pump. This pump has the advantage of replicating the flow without damaging particles within the fluid, such as those designed to replicate red blood cells. 
Gear pump based systems have been developed and refined over the last two decades. Petersen et al. [10] developed a dual gear pump system that relied on a computer-controlled valve to control the flow going to the test section. Hoskins et al. [4] explored using a signal-controlled gear pump to generate simple pulsatile waveforms. Plewes et al. [11] advanced the system by generating pulsatile flow using a similar design but improved computer waveform generation. This included the addition of a closed feedback calibration circuit to improve waveform reproduction. While Plewes' pump is simpler than the dualchamber pump, it requires electronics to carry out initial iterative calibration adjustments for each new waveform. Wong et al. [14] modified the design by introducing a software feedback loop using real-time velocity feedback from MRI scans.

The pumping system presented in this article was developed for generating pulsatile flows in flow phantoms using a variety of fluids (e.g., water, glycerin, isopropyl alcohol, and mixtures). This design allows users to optimize the flow parameters and tune the system for the needs of the experiment.

\section{Methods}

The pulsatile pumping system consists of a combination of a gear and a piston pump in series (Fig. 1). All components are mounted on a plate for easy access. The Teflon-coated stainless steel gear pump provides the steady mean component of the desired flow waveform. Unlike previous designs, the output gear pump does not oscillate, instead is operated under a constant load. The gear pump is preloaded with a back-pressure valve at its discharge. The back-pressure valve consists of a spring loaded diaphragm designed to apply a positive pressure to the flow in the event downstream pressures are greater than the gear pump outlet pressure. This ensures that the gears remain in constant contact to prevent reverse flow. For this experimental setup, an Oberdorfer Chemsteel S207 Pump (Oberdorfer Pumps, Syracuse, NY, USA) is used with a maximum throughput of $300 \mathrm{ml} / \mathrm{s}$. A Nikkiso HydroGarD back-pressure valve (Nikkiso Pumps, Houston, TX, USA) is placed at the discharge of the gear pump.

The piston pump, connected to the back-pressure valve, provides the oscillatory component of the flow waveform. It consists of a stainless steel, honed cylinder housing of bore $5.45 \mathrm{~cm}$ and a stroke of $10 \mathrm{~cm}$ containing a Delrin piston. The capped end of the cylinder has the flow ports. In order to achieve reverse output flow, the piston pump draws fluid at a faster rate than the gear pump outputs. The piston is driven by a servo motor attached to a Thomson

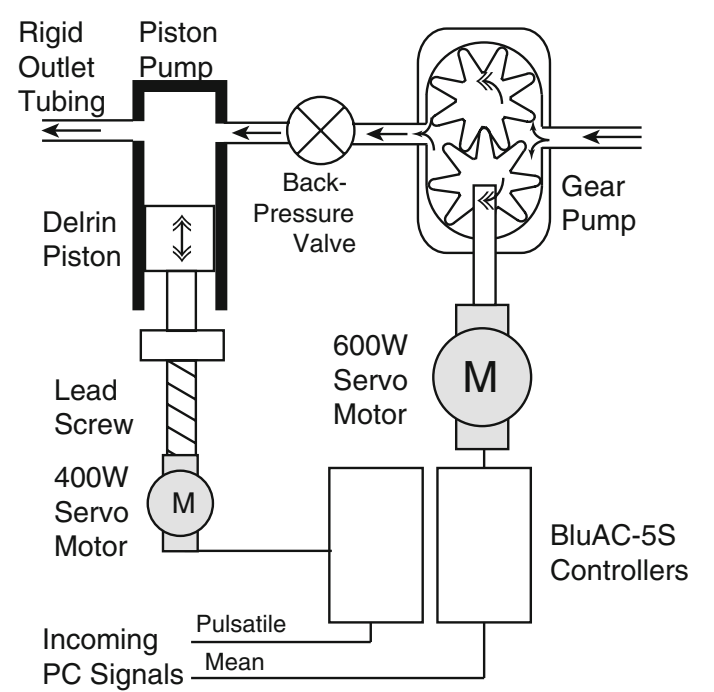

(a)

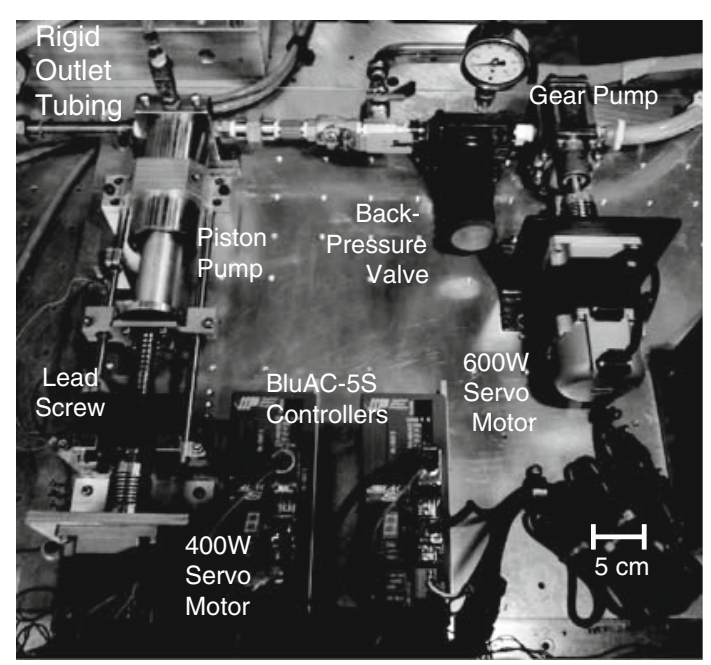

(b)

Fig. 1 Diagram (a) and photograph (b) of the gear-piston pumping system

Precision Plus Lead Screw with zero backlash (Thomson BSA, San Jose, CA, USA).

The gear pump is driven by an Applied Motion Products Alpha Series II 600W Servo Motor, with a rated maximum speed of $3000 \mathrm{rpm}$ (Applied Motion Products, Watsonville, CA, USA). The piston pump is driven by the $400 \mathrm{~W}$ version of the servo motor. Both motors connect to an Applied Motion Products BluAC5 controller, which include velocity control using an external signal. A single PC generates signals for both controllers through a Measurement Computing PCI-DAS6086 Data Acquisition Card (Measurement Computing, Norton, MA, USA). The gear pump is driven with a constant voltage signal to generate the mean flow, and the piston pump is driven with a variable voltage signal. For this experiment, the signals are generated by a $\mathrm{C}++$ code written to interpret the waveform input file. 
However, the system can easily be run from an analog signal generator or commercial packages such as Matlab, Labview, DASYLab, etc. with the appropriate hardware. Once connected with an experimental setup, the system must be tuned. The Quick Tuner Software, supplied by the controller vendor, is used to adjust the system gain settings to optimize the response. The software allows the user to measure the system's response to an input signal while a graphical user interface is used to adjust the gain settings until an acceptable response is achieved.

A simple closed flow loop is used for characterizing the system using distilled water. The pump is supplied by a reservoir at an elevation of $1 \mathrm{~m}$ to avoid cavitation at the inlet of the gear pump. Rigid copper tubing connects the pump outlet to the flow probe. It measures $57 \mathrm{~cm}$ long with two $90^{\circ}$ bends. Rigid tubing is used because compliant tubing may corrupt the waveform. Flow rates are measured by a Transonic $4 \mathrm{~N}$ ultrasonic flow probe in conjunction with a T206 flow meter (Transonic Systems Inc., Ithica, NY, USA). The flow probes have an accuracy of $\pm 5 \mathrm{ml} / \mathrm{min}$ and absolute accuracy of $\pm 7 \%$. Polyester-reinforced PVC tubing is used to connect the reservoir to the pump inlet and the flow probe outlet to the reservoir.

\section{Results}

A series of tests was conducted to evaluate the performance of the pumping system. The system frequency response is determined by measuring output amplitude (i.e., flow rate) to a fixed amplitude sinusoidal waveform with varying frequency from 1 to $8 \mathrm{~Hz}$. The accuracy and repeatability of the system are tested by measuring the output flow rate for a sinusoidal waveform as well as a measured coronary [9] and carotid waveforms [5]. A frequency of $0.5 \mathrm{~Hz}$ is used for the sinusoidal waveform tests, while a frequency of $1.0 \mathrm{~Hz}$ is used for the coronary and carotid waveform tests. Output data are recorded over 15 waveforms and compared to the input signal. In order to characterize the fidelity of the mean output to the input waveform, we use the root mean square deviation, $\sigma$, between the input signal and the mean output measurement.

Figure 2 shows results from a typical sinusoidal test case at $0.5 \mathrm{~Hz}$. The input signal (solid line) is plotted against the mean output signal (dashed line) and the upper and lower limits (dotted lines) of the set of 15 output waveforms. At this frequency, the mean output nearly matches the input waveform.

The frequency response to sinusoidal input is shown in Fig. 3. There are small variations in amplitude response in the $1-2 \mathrm{~Hz}$ range covering the frequency of most experiments modeling physiological blood flows. The frequency response shows a damped resonant frequency near $5 \mathrm{~Hz}$.

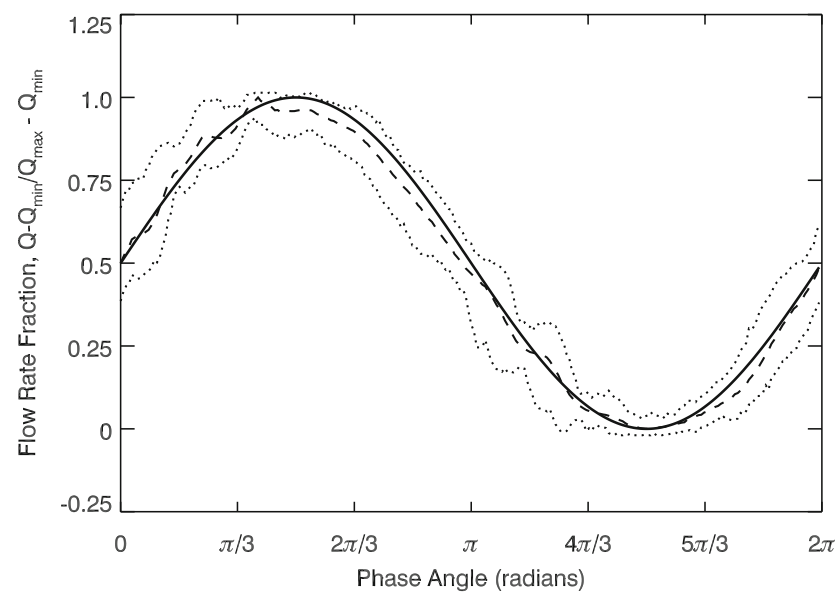

Fig. 2 Comparison of the sinusoidal input waveform (solid line), mean output flow (dashed line), and spread of the extrema (dotted lines)

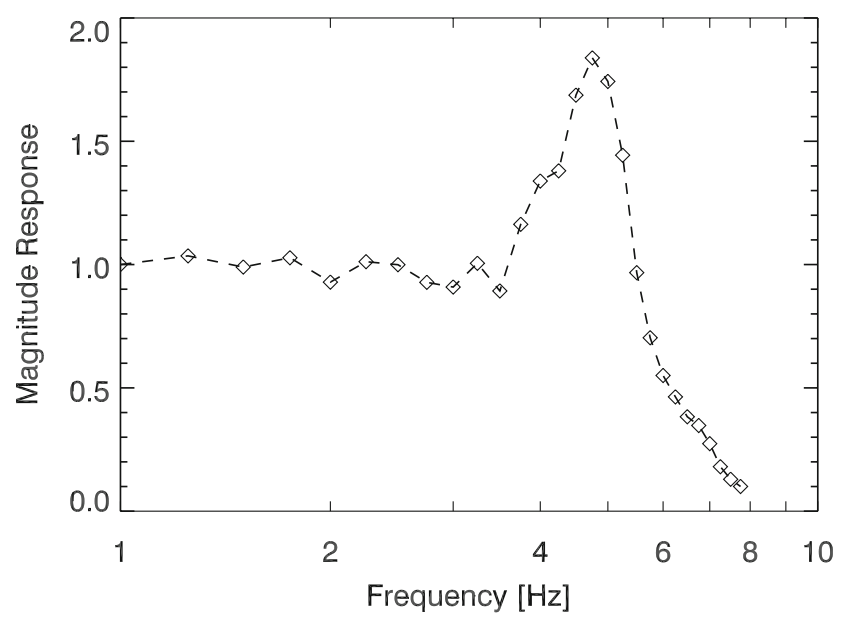

Fig. 3 Frequency response of the system

At higher frequencies, the amplitude decays rapidly, with the response falling to $28 \%$ of original amplitude at $7 \mathrm{~Hz}$ and less than $10 \%$ of original amplitude at $8 \mathrm{~Hz}$. The frequency response is dependent on the impedance of the downstream flow loop and the system components. As an example, changing the copper tubing to a shorter segment with no bends changes the peak response frequency to $\sim 3.5 \mathrm{~Hz}$. Because of this sensitivity to impedance, it is important to tuning and characterizing the system when connected to the experimental flow loop.

\section{Discussion}

The accuracy and repeatability tests display the performance of the system for physiological inputs: coronary (Fig. 4) and carotid (Fig. 5) waveforms. Root mean square deviations of the mean output flow curve to the sinusoidal, coronary, and 


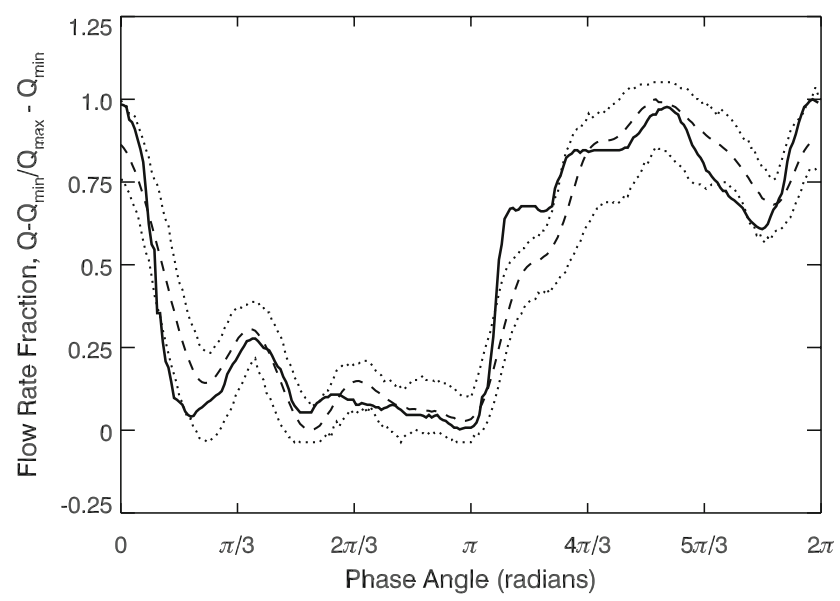

Fig. 4 Comparison of the coronary waveform (solid line) [9], mean output flow (dashed line), and spread of the extrema (dotted lines)

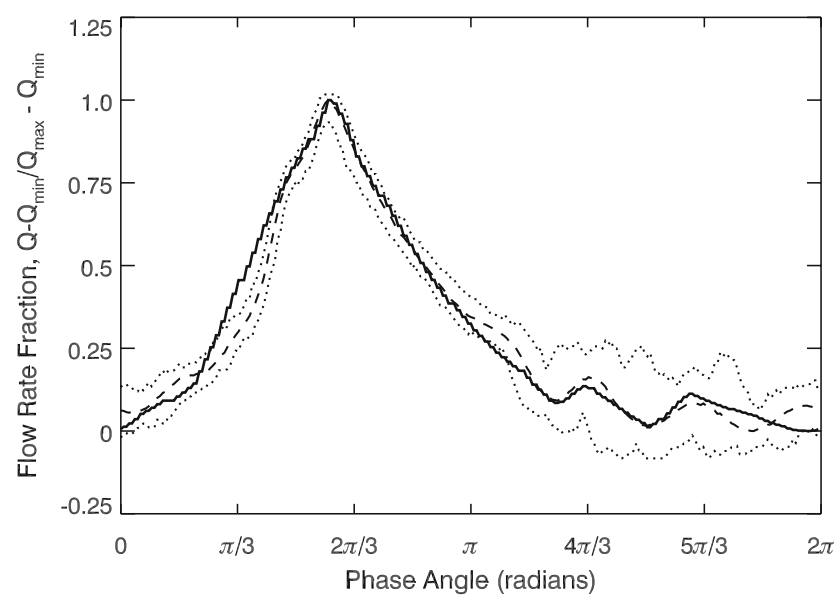

Fig. 5 Comparison of the carotid input waveform (solid line) [5], mean output flow (dashed line), and spread of the extrema (dotted lines)

carotid input waveforms are $\sigma=0.03, \sigma=0.10$, and $\sigma=0.05$, respectively. These results compare well to the performance of other physiological pumps. Frayne et al. [2] had an RMS deviation of $0.8 \mathrm{ml} / \mathrm{s}$ for a carotid waveform with a peak flow rate of $15 \mathrm{ml} / \mathrm{s}$. Plewes et al. [11] had a RMS error that was as high as $20 \%$ on the first iteration, but improved to less than $1 \%$ over 15 iterations for a $2-\mathrm{Hz}$ sinusoidal waveform. Wong et al. [14] were able to achieve aortic flow waveforms with a normalized cross correlations of 0.94 after the use of the pre-compensation scheme.

For a system without any feedback correction, the pump system is capable of replicating physiological waveforms. The greatest deviations occur when steep changes are present in the waveforms, such as in the coronary waveform. The mean, minimum, and maximum curves show a steep slope response, but the system is clearly unable to generate the instantaneous response requested. The data

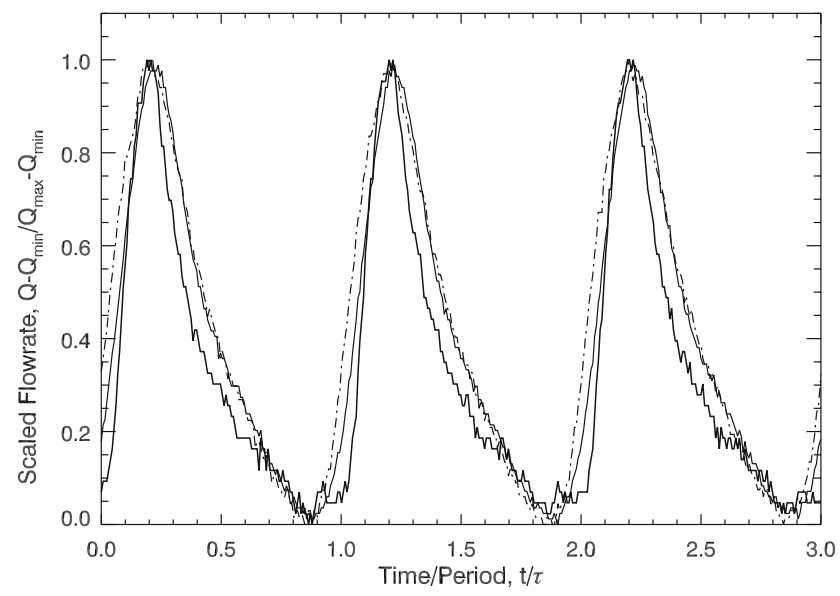

Fig. 6 Real-time phase adjusted flow output measurements for a basilar artery bifurcation experiment. The input waveform (solid line) drives the pump. The output flow is divided into two outlets (broken lines)

from the carotid waveform illustrate that this system has the least error at constant acceleration. The largest error can be seen in diastole during the small oscillations.

As application is in physiological modeling, Fig. 6 shows input and output waveforms from an experiment in cerebral aneurysms at the bifurcation of the basilar artery. The flow waveform, obtained from Kato et al. [6], is set to a frequency of $1.55 \mathrm{~Hz}$. The measurements from the system are obtained from flow meters placed at each of the outlet arteries and are phase shifted to adjust for lag introduced by the flow probes and motor controllers. Validation tests show good agreement between the input and outlet waveforms (Fig. 6). While the width of the outlet peak is wider than the input peak, the major features of the waveform are captured.

This article presents a pulsatile flow pumping system that can be easily adapted for most research applications requiring waveform reproduction, even if the waveform contains negative displacements. It is a continuous open control system which does not rely on feedback from flow meters. The components can be designed by the experimenter to meet requirements on the flow rate and amplitude; the simplicity of the design allows for the construction of a pump system for varying flow rates and working fluids. Through an interface using a software package of the experimenter's choosing, the system can directly replicate an input waveform, rather than relying on replicating the cardiac waveform and creating a flow loop with the appropriate impedance. Components be easily acquired commercially or machined in-house. The system does display variance in characteristics based on the impedance of the flow loop. In order to address this, the motors should be properly tuned upon installation. Once tuned, the system can replicate desired waveforms with reasonable accuracy. 
Future characterization experiments would compare the pumping system against the aforementioned designs in identical experimental setups. While a comparison of results are presented in this article, variations in the impedance of the setup make direct comparison imprecise. Future work on the pumping system would focus on isolating the sources of variation. While it is understood that the length of rigid plumbing affects system performance, the relationship of the length of the plumbing and flow disturbances (i.e., fittings, bends, wall roughness) to the variation may aid in optimizing the flow loop design. Exploring a software feedback system that uses the flow meter to optimize motor tuning may improve response time and reduce variations without affecting the input waveform.

Acknowledgments We gratefully acknowledge the contributions of Çağla Karaaslan, Duncan Maitland, Jason Ortega, Kathy Selby, Ward Small, Victoria Sturgeon, Gregory McCauley, and Wilson Tsai. This work was supported by the NIH (Grant R01HL067912).

Open Access This article is distributed under the terms of the Creative Commons Attribution Noncommercial License which permits any noncommercial use, distribution, and reproduction in any medium, provided the original author(s) and source are credited.

\section{References}

1. Eriksson A, Persson H, Linström K (2000) A computer-controlled arbitrary flow wave form generator. Rev Sci Instrum 71(1):235242. doi: $10.1063 / 1.1150189$

2. Frayne R, Holdsworth D, Gowman L, Rickey D, Drangova M, Fenster A, Rutt B (1992) Computer-controlled flow simulator for MR flow studies. J Magn Reson Imaging 2:605-612. doi: 10.1002/jmri.1880020522
3. Holdsworth D, Rickey D, Drangova M, Miller D, Fenster A (1991) Computer-controlled positive displacement pump for physiological flow simulation. Med Biol Eng Comput 29(6):565570. doi:10.1007/BF02446086

4. Hoskins P, Anderson T, Pelissier R (1989) A computer controlled flow phantom for generation of physiological doppler waveforms. Phys Med Biol 34:1709-1717. doi:10.1088/0031-9155/34/11/018

5. Jou L, Berger S (1998) Numerical simulations of the flow in the carotid bifurcation. Theor Comp Fluid Dyn 10:239-248. doi: $10.1007 / \mathrm{s} 001620050061$

6. Kato T, Indo T, Yoshida E, Iwasaki Y, Sone M, Sobue G (2002) Contrast-enhanced 2D cine phase MR angiography for measurement of basilar artery blood flow in posterior circulation ischemia. Am J Neuroradiol 23(6):530-542

7. Kheradvar A, Gharib M (2009) On mitral valve dynamics and it connection to early diastolic flow. Ann Biomed Eng 37(1):1-13. doi:10.1007/s10439-008-9588-7

8. Law Y, Cobbold R, Johnston K, Bascom P (1987) Computercontrolled pulsatile pump system for physiological flow simulation. Med Biol Eng Comput 25:590-595. doi:10.1007/ BF02441756

9. Ling S, Atabek H, Patel D (1975) Analysis of coronary flow fields in thoracotomized dogs. Circulation 37:752-761. doi: $10.1118 / 1.597504$

10. Petersen J (1984) Digitally controlled system for reproducing blood waveforms in vitro. Med Biol Eng Comput 22:277-280. doi: 10.1007/BF02442758

11. Plewes D, Urchuk S, Kim S, Soutar I (1995) An MR compatible flow simulator for intravascular pressure simulation. Med Phys 22:1111-1115. doi: 10.1007/BF0244275810.1007/BF02442758

12. Sadasivan C, Lieber B, Gounis M, Lopes D, Hopkins L (2002) Angiographic quantification of contrast medium washout from cerebral aneurysms after stent placement. Am J Neuroradiol 23:1214-1221

13. Segers P, Dubois F, Watchter DD, Verdonck P (1998) Role and relevancy of a cardiovascular simulator. Cardiovasc Eng 3(1): $48-56$

14. Wong P, Graves M, Lomas D (2008) Integrated physiological flow simulator and pulse sequence monitoring system for MRI. Med Biol Eng Comput 46(4):399-406. doi:10.1007/s11517-0080319-x 